\title{
A Novel Approach for Sustainable Product Development Education
}

\section{Mr. Lam F. Wong, Cuyahoga Community College, Metropolitan}

Lam Wong joined Cuyahoga Community College in 2012 as the Associate Dean of Engineering and Engineering Technology. He received a Bachelor of Science degree in Mechanical Engineering from Columbia University, a Master of Science degree in Mechanical and Aerospace Science from the University of Rochester, and a Master of Science degree in Electrical Engineering from the National Technological University. He has taught courses in engineering technology, operations management, and mathematics at three different institutions. Mr. Wong has extensive industrial experience in applied research, systems design, as well as leading large consumer and industrial product programs through all phases of the product development process.

\section{Henry Griffith, Wright State University}

Henry Griffith holds both a B.S. and Ph.D. degree from Wright State University in Electrical Engineering. In addition, he has obtained an M.S. degree in Management Science from the University of Dayton, as well as post-graduate certificates in Quality Assurance and Design of Experiments. He has 6 years experience as a full time research engineer working in the development of radio frequency systems for the defense industry. In addition, he has eight years experience in academia, including three years of full time instruction at Cuyahoga Community College in the Electrical Engineering Technology Department. 


\title{
A Novel Approach for Sustainable Product Development Education
}

\begin{abstract}
The classical principles of product development have been formulated through a framework that relies heavily upon assumptions of continuously declining validity in the modern marketplace. Mobile phones are one such product group that fall into the cycle of rapid consumption, disposal, and replacement. Such principles have traditionally led to a narrowly focused development approach, where systems and processes are optimized solely in terms of maximizing corporate profit. The recent increase in consumer demand for green products has counteracted this traditional approach, driving companies to adopt the triple bottom line or sustainability philosophy. The objective of this paper is to propose a uniform definition of Sustainable Product Development for utilization in academia based upon existing best practices in industry. The proposed definition is formulated as a coupling of an engineering process and a leadership framework. The sustainable product development engineering process has evolved significantly through years of continuous improvement upon the primitive design-build-test sequence. The sustainable product development leadership framework is generated by expanding the triple bottom line concept into seven core principles which form a sustainability vision for inspiring companies. The seven principles are - 1) Purpose, 2) Policy, 3) Process, 4) People, 5) Planet, 6) Product, and 7) Profit. Additionally, the paper discusses a strategy for implementing sustainable product development into engineering technology programs. The proposed strategy will rely upon an integrated learning pedagogy, in which the concepts are introduced in a technology orientation course and reinforced in a capstone product development course. Learning outcome assessment of this effort should guide the next phase of integrating sustainable product development in engineering technology curricula.
\end{abstract}

\section{Introduction}

Sustainable Product Development (SPD) is a modern necessity which integrates the concepts of sustainability into the product development process (PDP). While product development processes are well established, ideas of sustainability are relatively new and continuously evolving. The most primitive PDP relies upon iterations of the design-build-test sequence. Depending upon the complexity of their products, most companies typically adopt their own proprietary version of the PDP that is phase-gate driven. A critical aspect of this development is the establishment of the appropriate gate criteria that must be met prior to the exit of each phase.

Successful integration of sustainability into the PDP requires clear definitions of its core concepts. In light of this, many authorities have attempted to define the meaning of sustainability. The most widely quoted definition of sustainability came from the Brundtland Commission of the United Nations in 1987, stating that - "Humanity has the ability to make development sustainable to ensure that it meets the needs of the present without compromising the ability of future generations to meet their own needs ${ }^{1}$." More than two decades later, a group 
of scientists began to develop the measurable metrics of sustainability achievement, known as the Nine Planetary Boundaries, which are listed below ${ }^{2}$ -

- Climate change

- Ocean acidification

- Stratospheric ozone depletion

- Interference with the global phosphorus and nitrogen cycles

- Rate of biodiversity loss

- Global freshwater use

- Land-system change

- Aerosol loading

- Chemical pollution

Based upon its expanding importance in the modern marketplace, sustainability is becoming more prevalent in the operation strategies of various businesses. Companies are modifying their objectives from simply maximizing profit to a so-called "Triple Bottom Line" (TBL) mission. The TBL is formulated based upon social, economic, and environmental factors. Last year 3,208 companies worldwide and 944 companies in the U.S. were listed on the Dow Jones Sustainability Index ${ }^{3}$. This list consists of companies from a variety of industries, and includes well established manufacturers such as DuPont, which was established in 1802. In order to reflect its commitment to the culture of sustainability, DuPont has published the following statement on its website ${ }^{4}$

“As the world's population approaches nine billion by 2050, we face unprecedented challenges to sustainably address the critical needs of food, energy, and protection. Through our science-driven innovation and global collaboration, we create solutions that help meet these needs. Sustainability is at the core of what we do - from reducing our operational footprint, to developing renewable materials and safer products, to collaborating with others to create sustainable solutions. Our world is changing. The demographic shifts - about 150,000 more people on the planet each day - will increase urban growth and continue to place pressure on natural resources to feed the world, meet the growing energy demands, and keep people and the environment safe in a sustainable way. We recognize the value of long-term sustainable solutions to address the challenges. Around the world, we are working collaboratively to deliver scientific innovations and solutions along our value chains, with academics, governments, other companies and organizations.”

This is a compelling statement which demonstrates foresight towards the future challenges associated with the exponential growth of demand for finite resources. This outlook should not be surprising, as any company that has thrived for more than 200 years has clearly demonstrated a strong culture capable of long term sustainable growth.

As the importance of sustainability continues to increase in the modern economy, educational institutions at various levels have attempted to integrate its concepts into their curriculum. At the graduate level, courses in Environmental Policy and Sustainable Development have long existed 
in various Engineering and Engineering Management programs. Unfortunately, these courses were often electives placed late within the curriculum, thereby limiting the ability to integrate the concepts of sustainability throughout the program as desired. Based upon this deficiency, a conceptual framework based upon System Engineering techniques and Optimal Control Theory has been developed as a mechanism to reform engineering education in order to include aspects of sustainability across the curriculum ${ }^{5}$. In order to accommodate this increased focus on sustainability throughout the curriculum, the importance of faculty development within this area, including content training and learning competency standardization, has also been suggested in the literature ${ }^{6}$.

Numerous efforts have also been taken to teach the concepts of sustainability at the undergraduate level. For example, the Manufacturing Engineering Technology program at Ball State University has proposed an innovative curriculum teaching these concepts based upon an integrated learning pedagogy ${ }^{7}$. It should also be noted that the increased focus on the inclusion of sustainability in education is not isolated to colleges and universities, as recent developments have seen the proposed establishment of "Green Curricula" at the K-12 level ${ }^{8}$. Although these and similar efforts are promising, the importance of sustainability in the modern economy merits further investigation into best practices for optimizing its inclusion in educational curriculum.

The objective of this paper is to propose a uniform definition of Sustainable Product Development for utilization in academia based upon existing best practices in industry. The proposed definition is formulated as a coupling of an engineering process and a leadership framework, which respectively formulate the mission and vision for the process. The engineering process is based upon classic theories of product development integrated within a concurrent, sustainability focused framework. The leadership framework, which is developed as an expansion of the aforementioned TBL, completes the SPD process by providing guidance for the establishment of a sustainability focused culture within an enterprise. After introducing this process, a reflection detailing the challenges and opportunities associated with the inclusion of SPD principles within a two year engineering technology program is discussed.

\section{General Characteristics of Sustainable Product Development Processes in Industry}

Sustainable product development expands upon traditional product development by introducing additional considerations into the product development process. These additional factors include the environmental impact of source materials, as well as the end of life plan for the product. In addition, the environmental impact of the product during its usable life must also be taken into consideration in a SPD process. Based upon these additional considerations, product Life Cycle Analysis (LCA) and management have become an integral part of existing SPD strategies throughout industry ${ }^{9,10}$.

Environmentally conscious tools for manufacturing, management, and economics have also been used in the development of existing SPD strategies ${ }^{11}$. These efforts are based upon internal efforts, as well as concepts and best practices developed in government, academia, and nonprofit collaborations. For example in Table 1, the seven success factors for eco-efficiency, developed by the World Business Council for Sustainable Development, have had significant 
impact on the development of industrial SPD processes ${ }^{12}$. Three of these seven factors focus on the reduction of process outputs, while the remaining four focus on enhancement.

Table 1 - Seven Success Factors for Eco-efficiency

\begin{tabular}{|l|l|}
\hline Reduction & Enhancement \\
\hline$\bullet$ Material intensity of goods and services (RM) & $\bullet$ Material recyclability (ER) \\
\hline$\bullet$ Energy intensity of goods and services (RE) & $\bullet$ Sustainable use of renewable resources (ES) \\
\hline • Toxic dispersion (RT) & $\bullet$ Durability of products (ED) \\
\hline & $\bullet$ Service intensity of goods and services (EI) \\
\hline
\end{tabular}

Many companies have adopted the Eco-efficiency approach to their SPD process. The Remanufacturing Operation by Rank Xerox, Product Stewardship by DuPont, Responsible Care by Dow Chemical, Eco-efficiency by Ford Motor and DuPont, Evergreen Program by Interface Flooring Systems, By-product Synergy by Chaparral Steel, and LCA by AT\&T are some of the most commonly known company unique processes documented in case studies ${ }^{13}$.

\section{Proposed Engineering Process for Sustainable Product Development}

The proposed Sustainable Product Development process introduced in this paper results from the coupling of a sustainability oriented leadership framework along with a complex engineering process as shown in Figure 1. Most industrial engineering processes are much more intensive than the basic design-build-test cycle, which is often the only process with which engineering students are familiar. The proposed engineering process for the SPD concept is developed based upon proven industrial practices from companies like Xerox, Ford, and Plug Power. The remainder of this section summarizes the basic characteristics of each phase of the process.

It should be noted that the Sustainable Product Development Engineering Process (SPDEP) presented above is a specific instance of the well-known concurrent engineering framework. Concurrent Engineering is a concept that promotes cross-functional participation from all project stakeholders at the beginning of product development, as well as overlapping projects and tasks throughout the development process. Ideally, concurrent engineering based processes allow for the completion of a systems design in the shortest time possible.

As with any product development process, implementation of the SPDEP requires the preliminary establishment of the requirements of the target product. These attributes may be broadly characterized as relating to its form, fit, function, and finance. SPDEP is distinguished from traditional process development by its utilization of factors associated with the End of Life Strategy during the development of product requirements. In SPDEP, The End of Life Strategy involves the evaluation and selection of a series of so called "re-processing" options, such as repurposing, refurbishing, reusing, reconditioning, recycling, and reclaiming. In addition to the development of product requirements, the initial state of the SPDEP also involves Quality Function Development (QFD), as well as the development of the Systems Requirement Document (SRD). Both the QFD and SRD are essential aspects of nearly all existing industrial product development processes, and are thus well documented in the literature. 


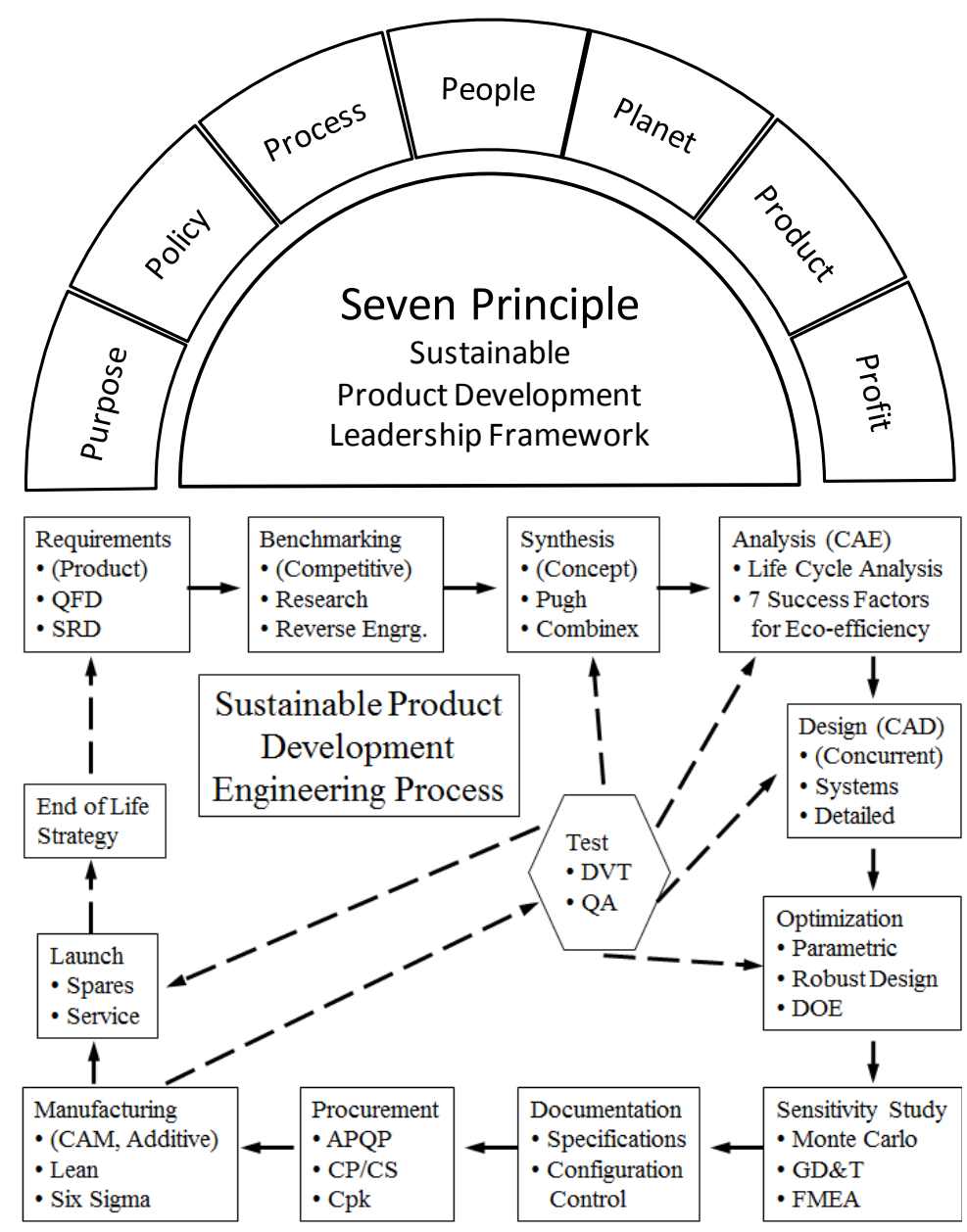

Figure 1 - Sustainable Product Development Leadership Framework and Engineering Process

After the requirements of the target product have been established, the SPDEP proceeds in a manner quite similar to other traditional product development processes. This is initiated by a Benchmarking phase, which may involve market research analysis, review of the literature, as well as physical teardown and reverse engineering. Next, concepts are developed and narrowed down by the Pugh or Combinex techniques in the Synthesis phase. The goal of this phase is to develop a so-called "Best of Breed" product, as determined based upon the results of the previous Benchmarking phase. Critical to the Synthesis phase is the consideration of interchangeability and commonality in product design. Ideally, the Synthesis phase may be exited after the development of a well-considered concept.

The concept developed in the Synthesis phase is naturally validated in the subsequent Analysis stage. It should be noted that modern engineering programs in many universities devote a significant portion of the curriculum to subject matters associated with the laws of physics. Component analysis is critical in order to ensure minimal iterations of any product design process. Located within the Analysis phase, Life Cycle Analysis and Seven Success Factors for Eco-efficiency are a central theme in the proposed SPDEP. The cost, energy, and toxicity involved in raw material extraction, and ultimately product disposal, are critical factors which must be estimated with high levels of accuracy during this phase. 
The following phase of Design has been historically characterized as a tedious, time intensive process. However, as has been well documented in the literature, technological advances in various CAD/CAM software packages have revolutionized this portion of product development. In addition, rapid prototyping technology now allows for the simplistic generation of concept parts for interim evaluation. Like the Analysis phase, concepts from the Design phase are also heavily emphasized in modern engineering curricula.

Another universal phase of the product design process which has been revolutionized by advances in computing is the Optimization phase. These advances have provided tremendous value to sustainability oriented product development processes, in which the basis of objectives is significant expanded relative to traditional product development. In the SPDEP, optimization parameters may be generated according to the previously identified factors of eco-efficiency and resource-efficiency. As is evidenced in the SPDEP flow chart, optimization should leverage each of the well-known techniques and algorithms established in the literature, including parametric optimization, robust design, as well as design of experiments.

The Sensitivity Study phase involves the utilization of well-established algorithms in order to analyze "what-if" type scenarios. As has been well documented in the literature, Monte Carlo simulation is very effective in studying random processes with multiple parameters. Geometric Dimensioning and Tolerancing (GD\&T), which should be implemented during the Sensitivity Study phase, is an essential part of any design process. GD\&T ensures the form, fit, and function of the product. Failure Mode Effect Analysis, which is also included in this phase, is a classical approach to achieve failure-prevention design or conduct post-mortem analysis.

After completing the Sensitivity Study phase, the SPDEP enters the Documentation phase, which is critical in order to ensure the success of the remainder of the process. The Documentation phase is a knowledge-capture process which maintains configuration control. Unfortunately, this process is rarely viewed favorably by development stakeholders, especially in light of the ever increasing speed of product development cycles. Next, the Procurement, or supply chain function, phase is implemented. This phase may occur at the raw material, part, component, or system level, depending upon the complexity and scope of the target product. The input of suppliers is critical during this stage of the SPDEP. In order to receive the best possible quality, cost, and deliver schedule of their orders, companies need to share openly essential information and to negotiate in good faith with suppliers ${ }^{14}$. Great companies strive to promote a highly collaborative business environment and are prepared to exchange ideas on sustainability best practices with their suppliers.

The subsequent Manufacturing phase encompasses the fabrication and assembly portion of the SPDEP. The previously discussed technological advances in rapid prototyping, namely in additive or direct digital manufacturing, allow for the efficient generation of either prototype or low volume production parts. In addition to rapid prototyping technologies, machine tools and classical manufacturing processes also remain critical in the fabrication of high volume parts. Innovations in manufacturing philosophies, such as lean six sigma, have also resulted in substantial efficiency improvements throughout manufacturing. Manufacturing theory and techniques have recently received a revival of interest throughout various engineering curricula, particularly at the two year engineering technology level. 
After manufacturing, the test phase of any product development process is crucial to ensure fulfillment of specifications. Two commonly known tests currently employed in various industrial environments are the Design Verification Test (DVT) and Quality Assurance (QA) test. Failure of the DVT typically results in the necessity that the design to go through an additional iteration of the development process. Failure of the QA test prevents the launch of the product into the marketplace.

The Launch phase of the process is an exciting event for all who are involved. As its preparation can make or break even a good product, the amount of planning required for this phase cannot be underestimated. Typically, an experienced team of launch specialists begins preparation months in advance of the launch date in order to plan for the build-up of product and spare parts inventory, warehousing strategies, marketing collaterals, as well as customer service and sales.

As was previously introduced, the End of Life Strategy phase is the distinguishing factor of any sustainable development process, including the SPDEP proposed herein. It examines "cradle to grave" implications along each step in the life of a product. The goal of efforts conducted within this phase is to minimize the environmental footprint of the product. Continuous improvement processes should be utilized here in order to turn wastes into resources. In addition, an effective End of Life Strategy should capture environmental lessons learned and track the stewardship of scarce resources.

\section{Proposed Leadership Framework for Sustainable Product Development}

While the key components of the engineering process for sustainable product development have been established for many years, the leadership framework aspect has recently received increased interest in light of marketplace changes. Perhaps the largest factor influencing this increased focus is the increasing demand for finite natural resources such as oil. The United States has benefited in the "cheap oil' era, especially during the period between 1985 and 2005. The GDP of the nation increased by $85 \%$ adjusted for inflation over this time period, while population increased by only 24\%. Additionally, the GDP per capita improvement was almost $50 \%$ over this period. Oil provides about $35 \%$ of the total energy used in the world and is responsible for many byproducts, such as rubber and plastic. Even today, it could be argued that oil is still relatively inexpensive. For purposes of illustration, Table 2 gives a comparison between gasoline and milk, two forms of liquid that are of significant interest to humans. Based upon this simplistic comparison, gasoline could easily be worth an order of magnitude more than milk.

Table 2 - Milk and Gasoline Comparison

\begin{tabular}{|l|l|l|}
\hline Key Attribute & Milk & Gasoline \\
\hline Sustainability & Renewable & Depletable \\
\hline Production & Steady & Peaking \\
\hline US Consumption in 2008 & $190[$ billion lbs/yr] & $2122[$ billion lbs/yr] \\
\hline Approximate Price Today & $\$ 3.50[$ per gal.] & $\$ 3.50[$ per gal.] \\
\hline Energy Density & $2.75[\mathrm{MJ} / \mathrm{Kg}]$ & $44.4[\mathrm{MJ} / \mathrm{Kg}]$ \\
\hline
\end{tabular}


Since the onset of the industrial revolution in the 1750s, the world population has sky-rocketed from 700 million to seven billion. World population is projected to reach nine billion by 2050 . This level of human and economic expansion is not sustainable, especially in light of the risk that peak oil production has been reached ${ }^{15}$. Figure 2 plots the annual global production of oil in giga-barrels against the total world population. The leveling effect can be seen from the fitted curve.

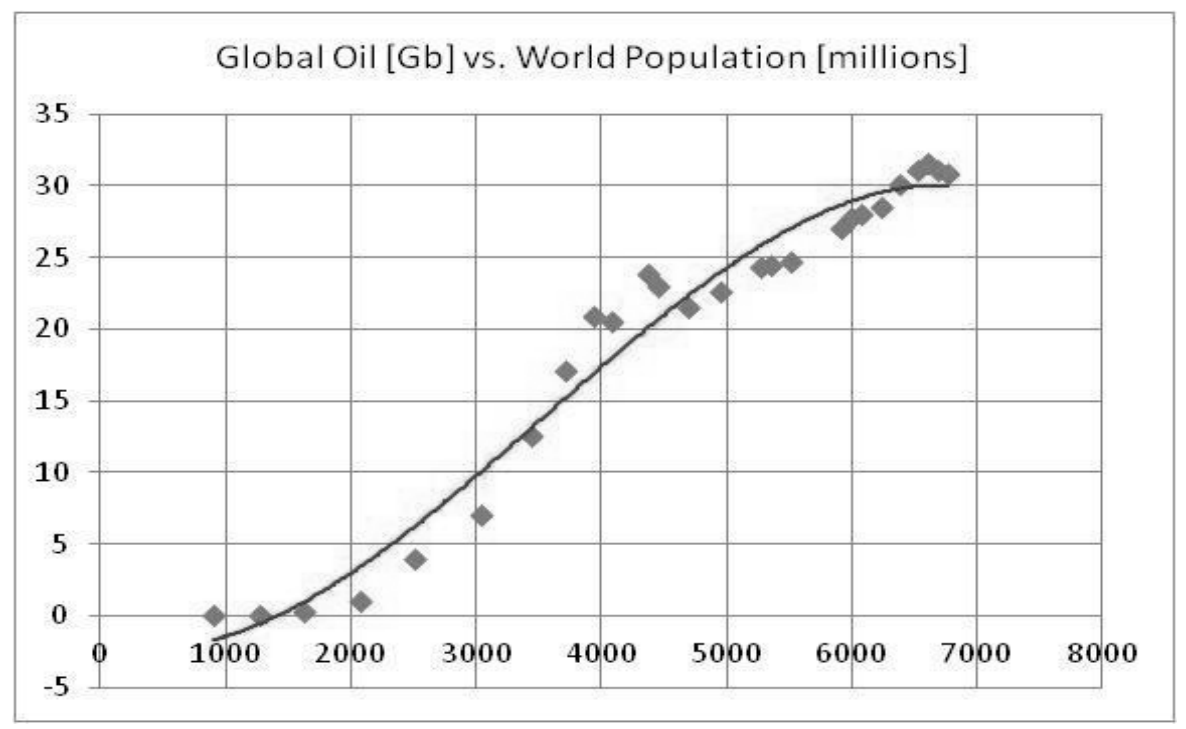

Figure 2 - Annual Oil Production vs. World Population

The leadership framework introduced herein proposes to address sustainable development for growth under limited resources. While the aforementioned engineering process provides the practical aspects of the proposed SPDEP, the leadership framework forms its philosophical counterpart. The proposed leadership framework is organized according to a set of Seven Principles (7P), which are - 1) Purpose, 2) Policy, 3) Process, 4) People, 5) Planet, 6) Product, and 7) Profit.

\section{Purpose}

The purpose of an organization provides an enterprise with a meaningful operational direction. In fact, one of the most common causes of organizational failure is the existence of multiple purposes whose trajectories conflict. When that occurs, the organization must examine their conflicting purposes against its vision. Visions should be forward looking in both long and short terms to anticipate the cadence of technological advances. Unfortunately, the vision of some companies is formed by looking at the rearview mirror.

Kodak is a prime example. Its founding purpose was to make photographs through photographic films. Its core competency has been in photosensitive chemicals and medium processing. Over the years Kodak grew and later decided to spin off the Eastman Chemicals Division in 1994, to fund its century old purpose. It adventured to become a pharmaceutical company by acquiring Sterling in 1988. The purpose of being a films and drugs company proved too diverse for the company to handle. Again in 1994 the company sold its non-imaging health-related businesses. Its ultimate dismay came from the digital photography the company invented in 1975. Kodak 
filed for Chapter 11 in 2012 because it failed to capitalize on the digital photographic technology and based its purpose and survival on the century old analog photographic technology ${ }^{16,17}$.

In order for all members of the organization to collaborate effectively, the purpose needs to be clear and articulated in a way that can be internalized by all stakeholders. In SPD, it is critical that the purpose of an enterprise be developed in the context of sustainability.

\section{Policy}

Policies are created to provide boundaries. While too many policies will stifle growth and creativity, too few will promote chaos. Policies are created to supplement the overarching purpose. In order to remain effective, policies must be tested and revised over time.

Empowerment of all stakeholders can be enabled by a well-established set of policies acting in conjunction with a well-defined organizational purpose. Like purpose, policies must also be crafted with sustainability in mind within the SPD process.

\section{Process}

Processes are a generic term for proven and captured knowledge which typically drive product quality. They are similar to policies, but are more technically oriented and are oftentimes implemented at lower levels of the organization. Good processes provide a competitive advantage to enterprises that often form a barrier to entry by potential competitors. Some of the most important processes are derived from trial and error, based upon lessons learned from prior experiences. Processes are critical in guiding the design and development of products or services.

\section{People}

People are arguably the most precious resource within an organization, as they provide the prerequisite creativity for sustainable development strategies. Unfortunately, finding talented people with integrity is one of the most difficult aspects of enterprise establishment. Companies owe their employees the opportunity for lifelong development according to Maslow's hierarchy of needs. An organized group of people having the same purpose can accomplish the most challenging task or design the most complex product. In a SPD leadership framework, it is critical that employees be exposed to a culture that promotes the principles of sustainability.

\section{Planet}

The role of the planet is indispensable, as it serves as the origin of all natural resources. While some resources required for product development are renewable, some unfortunately are not. In ancient times, a person used energy in the form of biomass primarily for food related activities. In industrialized countries today, a person uses more than 100 times the energy from mostly nonrenewable sources for food, housing/commerce, industry/agriculture, and transportation related activities $^{18,19,20}$. Renewable energy from the sun and moon in the forms of photovoltaic, solar thermal, hydroelectric, wind energy, and tidal wave power must be considered a part of the energy portfolio by companies wishing to implement SPD. 


\section{Product}

Products are the tangible outcomes of all companies, and are ultimately created for the improved quality of living and convenience of mankind. While consumers have historically ignored environmental impact as a factor during product selection, recent evidence suggests that this trend is in fact changing. When developing a broad sustainable product development framework, companies must balance several factors, including product life span, eco-efficiency, as well as the end of life plan. For purposes of illustration, Table 3 compares a spectrum of useful products according to similar criteria. Namely, five sustainability attributes are used to compare and evaluate each of the considered products.

Table 3 - Product Comparison

\begin{tabular}{|l|l|l|l|l|l|}
\hline Attribute & Toothbrush & Mobile Phone & PC & Automobile & Piano \\
\hline Life Span [yr] & $0.1-1.0$ & 1.5 & 4.5 & $7-20$ & 50 \\
\hline Usage & Consumable & Disposable & Extendable & Durable & Conservable \\
\hline Eco-efficiency & $\begin{array}{l}\text { 4 out of 7: } \\
\text { RM+RE } \\
\text { +RT+RM }\end{array}$ & $\begin{array}{l}\text { 3 out of 7: } \\
\text { RM+RE+EI }\end{array}$ & $\begin{array}{l}\text { 4 out of 7: } \\
\text { RM+RE } \\
\text { +ER+ED }\end{array}$ & $\begin{array}{l}\text { 5 out of 7: } \\
\text { RT+ER+ES } \\
\text { +ED+EI }\end{array}$ & 7 out of 7 \\
\hline End of Life & Repurpose & Reclaim & Recycle & Recondition & Refurbish \\
\hline Biodegradable & Poor & Poor & Poor & Fair & Good \\
\hline
\end{tabular}

Ironically, the above table emphasizes that products with increasing levels of technology oftentimes exhibit diminishing levels of sustainability. This paradigm represents one of the key challenges for SPD.

\section{Profit}

Profit is essential for the survival of all companies, and thus must still be a focus of any operational strategy. In a SPD context, it is critical that profit be reinvested in four of the 7Ps: Process, People, Planet, and Product. Newer manufacturing tools and processes are more efficient, thereby improving the achievement of sustainability metrics. Investment in employees allows for continuous professional development, also leading to improved organizational achievement. Community reinvestment has several positive effects, including the expansion of goodwill amongst both employees and consumers. Finally, by investing in the research and development of sustainable products, companies will better prepare themselves to compete in a marketplace which is increasingly focused on sustainability.

\section{Challenges of Integrating SPD in Engineering Technology Curricula}

As was discussed in the introduction of this paper, there has been significant effort expended to include content from SPD into various forms of engineering curriculum. While the efforts have been distributed across the various levels of higher education, few efforts have explicitly focused upon the challenges and potential rewards of integrating such content into two year engineering technology programs at community colleges. Such integration is especially important for colleges within regions whose economies rely significantly upon manufacturing. 
One such institution is Cuyahoga Community College (Tri-C), which is located in Cleveland, Ohio. Currently, the College offers several different degrees in engineering technology (Electrical/Electronic, Biomedical, Construction, and Mechanical) with one specifically focused in manufacturing concepts (Manufacturing Industrial Engineering Technology, or MIET). As is the case with many curriculums in technology, the pace of advances in industry has exceeded that of coursework modification. Therefore, appropriate initiatives have recently been taken in order to invest in the redevelopment of existing coursework to include the aforementioned aspects of advanced manufacturing technology.

Courses were also introduced into the MIET program within the last decade to emphasize the basic concepts of Industrial Design. In spite of this relatively recent addition, significant modifications are also required within these courses in order to incorporate the impact of sustainability concepts in the modern economy. Unfortunately, the pace of content modification is extremely limited in two year engineering technology programs, due to both the significant instructional workload on faculty, as well as a lack of funding to provide either release time or required equipment.

Perhaps the most significant challenge of integrating new content into a two-year engineering technology curriculum is the limited amount of degree credit hours available. This is especially significant in light of recent modifications to funding in higher education within Ohio, which shifts the funding metrics to degrees granted as opposed to enrollment. In light of this modification, many programs have attempted to reduce the number of credit hours contained, thereby making the addition of new content challenging. One potential solution to this problem currently being investigated at Tri-C is the development of post-graduate certificates. While this mechanism has been common at the graduate level, it is relatively new at the community college level.

Another substantial barrier to integrating the concepts of SPD into educational curriculum is the fact that some concepts are simply not well suited to be addressed in a classroom environment. This phenomenon further emphasizes the importance of internship and co-op experience for students. Recently, Tri-C has devoted significant attention to the betterment of the co-op program, reorganizing a College-wide office with the responsibility of establishing collaboration with industry and identifying opportunities for students. In addition to College-wide efforts, departmental faculty and administration are also committed to building such industrial partnerships.

\section{Opportunities for Implementation Strategy of Integrating SPD in Technology Programs}

Potential solutions to addressing these challenges include collaboration with both internal and external partners, as well as the procurement of external funding. Recently, Tri-C has demonstrated a commitment to each of these strategies. Namely, in late 2012, Tri-C applied for and has since received 2.5 million dollars of external funding through the United States

Department of Labor in support of retraining workers impacted by global trade. This grant is not only significant due to the amount of funding, but also in its mission to increase collaboration between the academic offerings of the College and its workforce development. 
The title of the project is "Manufacturing Product Development Associate Degree and Workforce Development Project." The objective of the project is to develop and enhance the program of study with innovative strategies. A portion of the funding will be used initially to incorporate sustainability concepts in an introductory course titled "Technology Orientation." The course content development and delivery work has begun in real time.

Subsequently, in-depth sustainability principles as discussed in this paper will be integrated into an existing capstone course "Fundamentals of Products Development and Manufacture." This approach to integrate sustainability concepts in existing coursework will serve as a pilot to teach SPD without increasing the number of credit hours for the program. Learning outcome assessment of this pilot effort should guide the next phase of integrating SPD in engineering technology curricula.

\section{Conclusions}

Sustainable product development is a necessity for companies wishing to compete in the modern economy. While product development processes with a sustainable focus are being implemented in industry, little effort has been devoted to developing a generalized theory of SPD suitable for integration within educational curriculum. The generalized SPD proposed herein consists of both an engineering process and leadership framework, which is well suited for demonstrating to students the broad scope of SPD within a modern enterprise. In spite of the urgency to integrate SPD into educational curriculum, various constraints have limited its inclusion, especially at the community college level. This is especially troublesome for colleges located within areas that are reliant on manufacturing, such as Cleveland, Ohio, and other so-called "rust-belt" regions. Regardless of these challenges, Cuyahoga Community College has taken initiative to modify existing coursework in order to include the essential concepts of SPD. Further efforts are required in order to develop curriculum capable of providing value to local manufacturers.

\section{Acknowledgement}

The authors would like to acknowledge Roger Saillant, Ph.D., Executive Director, Fowler Center for Sustainable Value, Weatherhead School of Management at Case Western Reserve University. He is a recognized authority in sustainability and holistic management techniques. The authors were inspired by Dr. Saillant in many of the sustainability concepts discussed in this paper.

\section{Bibliography}

[1] United Nations General Assembly (1987). Report of the World Commission on Environment and Development: Our Common Future. Transmitted to the General Assembly as an Annex to document A/42/427 - Development and International Co-operation: Environment.

[2] Rockström, Johan (2009). Planetary Boundaries: Exploring the Safe Operating Space for Humanity, Ecology and Society, Vol.14, No. 2, Art. 32. 
[3] The Dow Jones Sustainability Indices, as Benchmarks for Investors who Integrate Sustainability Considerations into their Portfolio. http://www.sustainability-indexes.com/

[4] DuPont Sustainability Section of Company Website. http://www2.dupont.com/inclusive-innovations/enus/gss/sustainability.html

[5] Czuchry, Andrew; Oarker, Michael; and Bridges, Robert (2010). Achieving Organizational Sustainability: An Engineering Management Challenge or Opportunity? American Society for Engineering Education, AC 2010-60.

[6] Zhang, Qiong; Vanasupa, Linda; Mihelcic, James R.; Zimmerman, Julie Beth; and Platukyte, Simona (2012). Challenges for Integration of Sustainability into Engineering Education, American Society for Engineering Education, AC 2012-4565.

[7] Kanu, Rex C.; Betz, Pamela Elizabeth; and Cotton, Samuel (2012). An Approach to Incorporating Sustainability in a Manufacturing Engineering Technology Program, American Society for Engineering Education, AC 2012-4677.

[8] Darwish, Muge Mukaddes; and Agnello, Mary Frances (2009). Sustainability or Green: Challenges and Changes for Educators and the Engineering Curriculum, American Society for Engineering Education, AC 2009-1794.

[9] Gregg, Michael H. (2002). Environmental Life Cycle Analysis for Engineers, Proceedings of the 2002 ASEE Annual Conference \& Exposition, Session 3251.

[10] Richter, David; McGinnis, Sean; and Borrego, Maura (2007). Assessing and Improving a Multidisciplinary Environmental Life Cycle Analysis Course, ASEE 2007, AC 2007-1826.

[11] Choi, Jun-Ki; Stuart, Julie Ann; and Ramani, Karthik (2003). Decision Support Tools for Environmental Product and Process Management: Survey and Needs, Environmental Informatics Archives, Vol.1 (2003), 2437EIA03-004.

[12] Sustainable Development and the Role of Eco-efficiency, Innovation and Corporate Social Responsibility. http://www.wbcsd.org/home.aspx

[13] Allocca, Camille (2000). Eco-Efficiency and Sustainable Product Development Sustainable Energy. web.mit.edu/10.391J/www/proceedings/Allocca2000.pdf. http://web.mit.edu/10.391J/www/proceedings/Ecoefficiency_Allocca2000.pdf

[14] Wong, Lam (2013). A Generalized Learning Curve Adapted for Purchasing and Cost Reduction Negotiations, Advances in Operations Research, vol. 2013, Article ID 584762, 9 pages, 2013. doi:10.1155/2013/584762.

[15] Schellnhuber, Hans Joachim (CBE Chair) (2011). World in Transition A Social Contract for Sustainability, German Advisory Council on Global Change.

[16] Mui, Chunka (2012). How Kodak Failed. http://www.forbes.com/sites/chunkamui/2012/01/18/how-kodakfailed/

[17] Kodak Company Website. http://www.kodak.com/ek/US/en/Home.htm

[18] de Sousa Jr., Paulo T. (2003). The Ethanol and Biodiesel Programmes in Brazil: A Brief Discussion, Joint UFMT-UNU Pantanal Regional Environmental Programme.

[19] Goldemberg, J; Johansson, TB; Reddy, AKN; and Williams, RH. (1985). Basic needs and much more with one kilowatt per capita. Ambio 1985, 14:190-200.

[20] De Martino Jannuzzi, Gilberto and Goldemberg, Jose (2012). Has the situation of the 'have-nots' improved? Wires Energy Environ 2012, 1; 41-50 doi: 10.1002/wene.20. 